\title{
A portable dual-beam photostimulator
}

\author{
LUIS M. PROENZA \\ Vision Research Laboratory, Department of Zoology, University of Georgia, Athens, Georgia 30602
}

\begin{abstract}
The construction of a compact, portable, dual-beam photostimulator of Maxwellian-view design is described. The instrument has ample parametric stimulus control, with provisions for added components or expansion, and is suitable for either psychophysical or electrophysiological applications.
\end{abstract}

Psychophysical and electrophysiological studies of visual function face demanding requirements for precise parametric stimulus control. Short of developing specialized photostimulators for individual applications (e.g., Baron, 1973; Brown, 1964; Talbot \& Kuffler, 1952), the usual approach is to rely on the flexibility of the triangular aluminum optical bench with its modular accessories and relatively low cost. However, when dual or multiple optical paths are involved, adjustments are frequently cumbersome, and size and the possibility of misalignment make it impractical to move the photostimulator or even to routinely change its use from psychophysical to electrophysiological applications or vice versa. A compact and easily portable photostimulator is also highly desirable when collaborative research is planned with colleagues lacking optical instrumentation or when the system's specialized features can enhance instructional demonstrations.

This paper describes a dual-beam photostimulator of Maxwellian-view design. The construction features were developed under the following constraints: (1) The system had to be small in size and lightweight so as to be easily portable; (2) parametric stimulus flexibility had to be sufficient for most psychophysical or electrophysiological applications; (3) the system should be easily modifiable to include additional components or needed expansion; (4) controls had to be readily accessible in the vertical plane; and (5) the system was to have a cost comparable to or lower than that of optical bench-plus-components systems. Although these design criteria can be met with commercially available miniature components, the cost factor is much greater than that achieved in the system described in this paper. Only general construction and component details are given here, so that users can more readily try to adapt

The system discussed in this paper was influenced by that originally designed by Harris Ripps (see Dowling \& Ripps, 1971). I am indebted to him for discussions concerning his design. Robert F. Miller stimulated this project by suggesting the benefits of vertically arranging optical benches and controls. I thank Ralph E. Morton for his help in overseeing the construction of the photostimulator and Chester J. Karwoski for helpful suggestions concerning the manuscript. This work was supported by NIH Grants EY-00973, EY-03526 1 K04 EY-00042, and 71 K04 EY 00218. their existing optics. Details of specific components are available, if desired.

\section{DESIGN AND CONSTRUCTION}

Figure 1 shows a schematic diagram of the photostimulator. It consists of a single light source feeding two identical achromatic lens pathways, each incorporating interference (IF) or neutral density (NDF) filters and wedges, apertures mounted on positioning devices, and electromagnetic shutters. Considerable flexibility is thus available for stimulus control, and more can be readily gained by the addition of other simple accessories.

\section{Housing}

Acting as an optical "bench" in the system's design is a rectangular $40 \times 60 \times 15 \mathrm{~cm}$ welded aluminum housing characterized by (1) circular end apertures of $4.5-\mathrm{cm}$ diameter that define the axis of four primary optical paths along the sides of the housing, (2) slotted channels parallel to the center of each path intended to carry modular components (lens holders, apertures, shutters, etc.), and (3) corner braces designed to permit mirrors or beam splitters to be inserted at the intersection of any two paths. Note that the end apertures can provide alternate exit ports for the light output or can serve as entry ports for other light sources or when two housings are used together to form expanded systems.

The housing is constructed from .25 -in. (sides) and .5 -in. (center partition) aluminum plate, cut to size and milled for the slotted channels and end apertures. The ends may be rabbeted to facilitate alignment, and miter clamps are used to hold the pieces rigidly in position during welding. Four pairs of corner braces, spaced laterally, to accept the mirror mounts are then also welded into place. If desired, the housing may alternatively be assembled with machine screws.

\section{Optical Path}

Identical achromatic lens pathways are used for both beams of the photostimulator (Figure 1). In each case, a short focal length (fl) lens $(66 \mathrm{~mm})$ placed in front of the lamp filament first collimates the light that is to pass through filters (NDF or IF) and wedges (W), and another short $\mathrm{fl}$ achromat $(66 \mathrm{~mm})$ then images the lamp fila- 


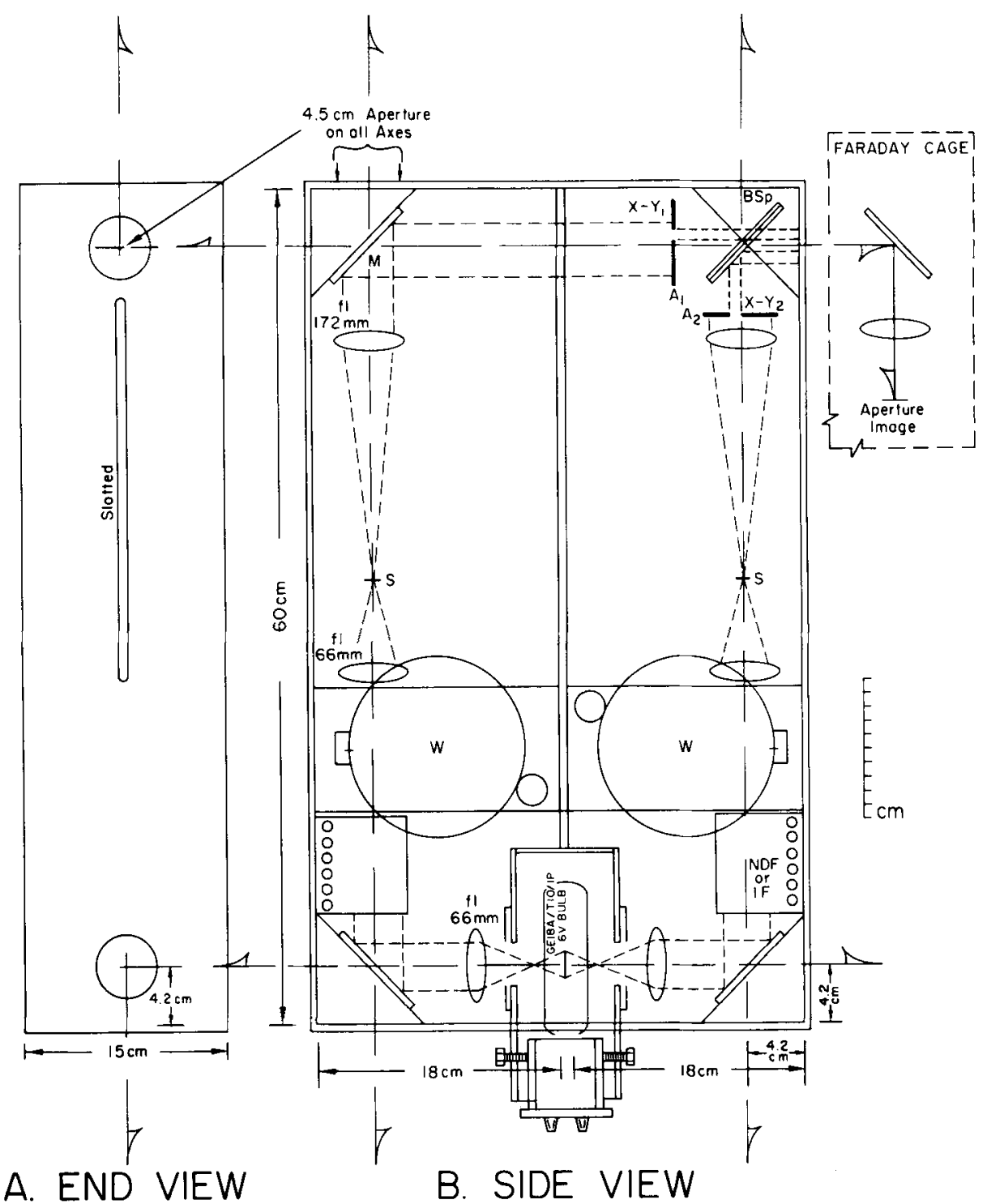

Figure 1. A portable dual-beam photostimulator: Schematic diagram of main construction details and dimensions. (A) End view, showing end apertures and slotted channels. (B) Side view, showing major details and components of the housing: $M=$ front surface mirrors mounted between corner braces; $X-Y_{1}$ and $X-Y_{2}=$ positioning devices; $A_{1}$ and $A_{2}=$ apertures; $S=$ shutters; $W=$ neutral density wedges; $N D F$ and $I F=$ neutral density and interference filters; $B S p=$ beam splitter. Output of the system is shown directed into a Faraday cage and through a final lens to create an image of the apertures at the plane of the retina.

ment at the plane of the shutters (S). Light is again collimated by a longer fl lens $(172 \mathrm{~mm})$ before output from the housing. Both beams are made collinear by a beam splitter (BSp) and directed to a final lens, outside the housing, which images field apertures $A_{1}$ and $A_{2}$ onto the plane of the retina and a monitor screen.

\section{Accessories}

Various types of modular accessories are required in order to mount optical components and to achieve stimulus control. Many of these can be obtained commercially and adapted to the dimensions of the photostimulator, or alternatively, some can be readily con- structed and may provide added conveniences or reduced cost.

Mirror mounts. Front-surface mirrors are clamped or glued to spring-loaded mirror mounts carried on a rectangular aluminum base plate. Three adjustment screws on the base plate control mirror alignment, and locating pins on one side and a tapped hole opposite serve to secure the base plate to the corner braces. In practice, the base plate is slipped between a pair of corner braces until the pins seat in matching locating holes. It is then lowered until the tapped hole accepts a machine screw inserted through the opposite corner brace. After the base plate is thus secured, the adjust- 
ment screws that align the mirror can be easily reached through adjacent end apertures in the housing.

Lens holders. Circular lens holders are mounted on short pillars so that their center coincides with that of the optical axis. The base of the pillar may be milled to fit the slotted channels at right angles to the lens holder, and it accepts a retaining screw used to position lens holders anywhere along the lengths of the channels. Note that if tubing of a suitable outer diameter is used to construct the lens holders $(8.4-\mathrm{cm}$ outer diameter, $4.2-\mathrm{cm}$ radius per Figure 1), a pillar is not needed and the rim of the holder is then tapped to accept the retaining screw. In this case, the sides of the slotted channel tend to exert a self-aligning effect on the lens holder.

Apertures and positioning devices. Any suitable microscope or micrometer $x-y$ stage (with at least a millimeter scale and vernier) may be easily modified to support an iris diaphragm aperture. These positioning devices, like the lens holders, are mounted on carriers that permit them to be moved and secured along the length of the channels and positioned at a plane conjugate with that of the retina. The carriers must be proportioned so that the midpoints of the $\mathrm{x}$ and $\mathrm{y}$ movements, and thus the center of the iris diaphragm, are aligned with the optical axis.

A simple and inexpensive way of generating annuli and other stimulus patterns is to rely on the rim of the iris diaphragm for locating various inserts. These inserts can be made from thick-wall aluminum tubing machined for a slip-fit to the inside rim of the iris diaphragm; stimulus patterns are made on 25 -mm-diameter glass cover slips (Corning), which are then attached to the inserts. Annuli, for example, can be generated by making a series of increasing-diameter black spots on the cover slips and carefully centering and gluing them to the aluminum inserts. Once in place, one need only adjust the iris diaphragm aperture to change annulus outer diameter or exchange inserts to alter inner diameter.

Neutral density or interference filter boxes. Rapid exchange slide carriers, which permit filters to be brought in and out of the light path as needed, can be incorporated into a box to accommodate several $2 \times 2$ in. glass filters. If the wedges are not used, a box accepting a larger number of filters than that shown in Figure 1 can be made.

Balanced neutral density wedge holders. Figure 2 shows the design characteristics of the wedge holders used for the photostimulator. In principle, they are similar to those that are available commercially, except that (1) an offset, right-angle drive is used to permit bottom or side mounting, and (2) the construction of the box allows the wedges to be sealed, except for an optical path, thereby reducing the accumulation of dust and debris on the delicate wedge surfaces. A gear ratio of 10:1 is used to couple the control knob to both the indicator and the wedges. A worm gear drives the vertical shaft, which, in turn, operates upon a pair of

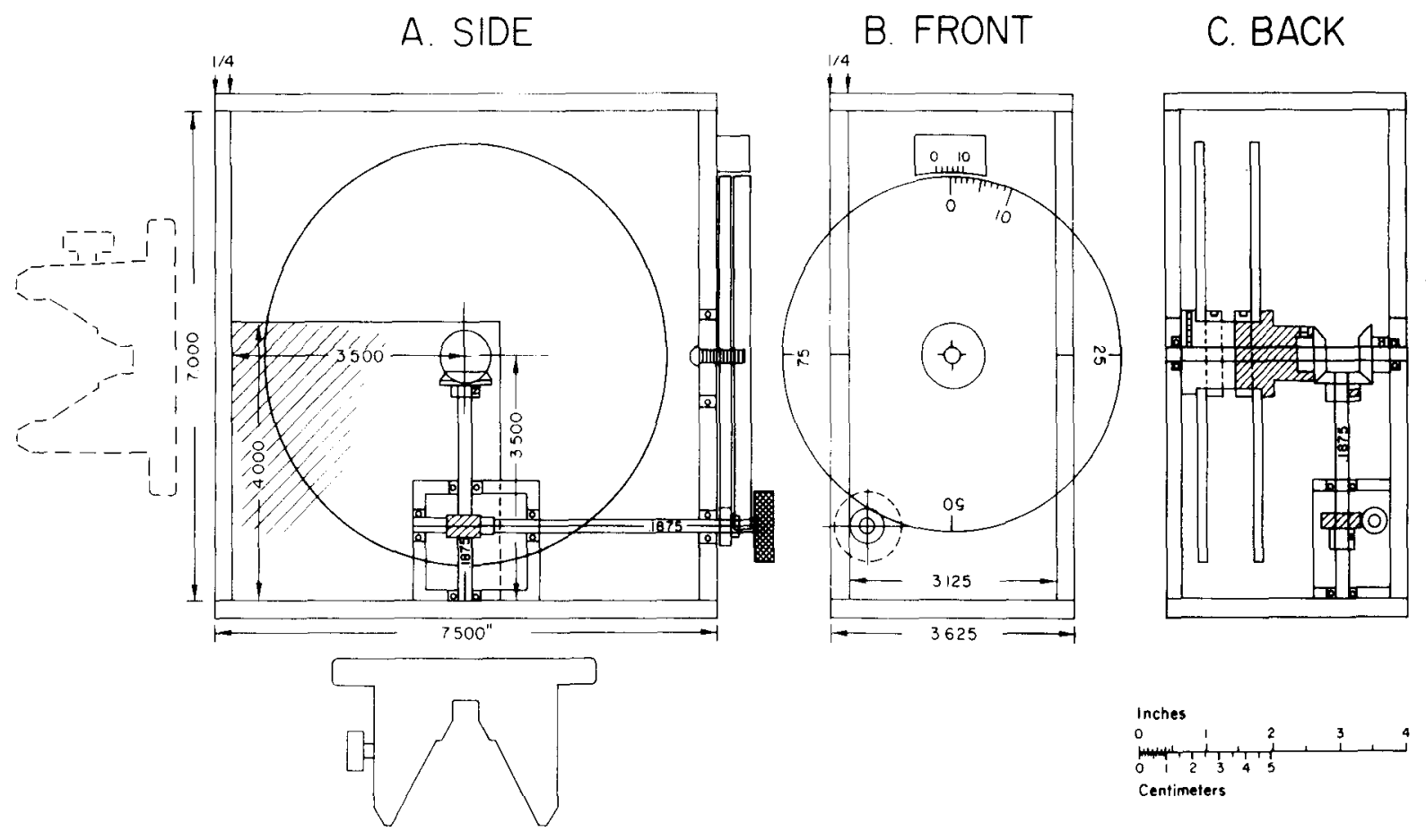

Figure 2. Balanced neutral density wedge holders for vertical or horizontal mounting: Schematic diagram of main construction details and dimensions. A, B, and C, respectively, are side, front, and back views of the holder. Note optional location of mounting base. (See text for discussion.) 
bevel gears to effect opposite directions of rotation for the two wedges.

Shutters. Dual-leaf electromagnetic shutters with a $25 \cdot \mathrm{mm}$ aperture are used in the system. They are secured to the rim of a standard lens carrier (see above) and placed at a filament image to optimize stimulus rise time.

Light source. A tungsten ribbon filament lamp (GE $18 \mathrm{~A} / \mathrm{T} 10 / 1 \mathrm{P}, 6 \mathrm{~V})$, driven at $17 \mathrm{~A} \mathrm{dc}$, feeds both optical paths from a machined, ribbed aluminum, cylindrical housing cooled by convection. Apertures on opposite sides of the lamp housing coincide with the optical axis of the photostimulator and bear holders for IR and UV filters. The lamp base is bolted to an aluminum cylinder that slides into the lamp housing from below and allows the lamp filament to be centered with respect to the optical axis of the system. In the configuration shown in Figure 1, the maximum retinal illuminance attainable is about $7,500 \mathrm{~mm} / \mathrm{m}^{2}$.

Other. The required electronics (shutter time, shutter and lamp power supplies, and ammeter) may be housed together in a small, portable relay rack. Final system lenses, suitable to the application requirements for the photostimulator, may be attached through a carrier arm fixed directly to the photostimulator, or they may be left in place at a specific application site.

\section{REFERENCES}

Baron, W. S. Maxwellian view stimulator for electrophysiological or psychophysical work. Applied Optics, 1973, 12, 2560 . 2562.

Brown, K. T. Optical stimulator, microelectrode advancer, and associated equipment for intraretinal neurophysiology in closed mammalian eyes. Journal of the Optical Society of America, 1964, 54, 101-109.

Dowling, J. E., \& Ripps, H. S-potentials in the skate retina: Intracellular recordings during light and dark adaptation. Journal of General Physiology, 1971, 58, 163-189.

TAlBot, S. A., \& KufrLER, S. W. A multibeam ophthalmoscope for the study of retinal physiology. Journal of the Optical Society of America, 1952, 42, 931-936.

(Received for publication March 22, 1982; revision accepted May 7, 1982.) 